\title{
LAS FAUNAS DE REPTILES MARINOS DEL CRETÁCICO FINAL DE EUROPA (MARGEN NORTE DEL TETIS MEDITERRÁNEO)
}

\author{
Nathalie BARDET' y Xabier PEREDA SUBERBIOLA \\ ' Laboratoire de Paléontologie des Vertébrés, Université Pierre et Marie Curie, URA 1761 \\ du CNRS, Case 106, 4, place Jussieu, 75252 París cedex 05, Francia. \\ 2 Universidad del País Vasco / Euskal Herriko Unibertsitatea, Fac. Ciencias. Dpto. \\ Estratigrafía y Paleontología. Apdo. 644. 48080 Bilbao.
}

Bardet, N. y Pereda Suberbiola, X. 1996. Las faunas de reptiles marinos del Cretácico final de Europa (margen norte del Tetis mediterráneo). [Marine reptile faunas from the latest Cretaceous of Europe (northern margin of the Mediterranean Tethys)]. Revista Española de Paleontología, 11 (1), 91-99. ISSN 0213-6937.

\begin{abstract}
Latest Cretaceous (Santonian to Maastrichtian) marine reptile faunas from the northern margin of the Mediterranean Tethys are analysed from taxonomic and biostratigraphic points of view. A correlation is established between the main fossiliferous units and the belemnite zones. Marine reptile faunas from the European province between $30^{\circ}$ and $40^{\circ} \mathrm{N}$ are dominated by mosasaurs and chelonioid turtles. The plesiosaurs (both elasmosaurs and pliosaurs) are quite rare and occur in the more northerly localities (above $40^{\circ} \mathrm{N}$ ). Dyrosaurid crocodiles and palaeophid snakes are unrepresented in the European fossil record. This distribution pattern of marine reptiles could be related to differences in latitude.
\end{abstract}

Keywords: Marine reptiles, mosasaurs, turtles, plesiosaurs, Late Cretaceous, European province, biostratigraphy.

\section{RESUMEN}

Se analizan las faunas de reptiles marinos del Cretácico final (Santoniense a Maastrichtiense) de Europa, en el margen norte del mar de Tetis mediterráneo, desde un punto de vista sistemático y bioestratigráfico. Se establece una correlación entre las principales formaciones y las zonas de belemnites. Las faunas de reptiles marinos de la provincia europea situadas entre los $30^{\circ}$ y $40^{\circ} \mathrm{N}$ de latitud están dominadas por los mosasaurios y las tortugas Chelonioidea. Los plesiosaurios (elasmosaurios y pliosaurios) son bastante escasos y provienen de las localidades más septentrionales (a partir de $40^{\circ} \mathrm{N}$ ). Los cocodrilos Dyrosauridae y las serpientes Paleophidae no están representados en el registro fósil europeo. Este modelo de distribución de los reptiles marinos parece estar controlado por los gradientes latitudinales.

Palabras clave: Reptiles marinos, mosasaurios, tortugas, plesiosaurios, Cretácico final, provincia europea, bioestratigrafía.

\section{INTRODUCCIÓN}

Los reptiles fueron un componente importante de los ecosistemas marinos del Mesozoico en tanto que depredadores situados en la cima de la pirámide trófica. Desde el Triásico Inferior al Cretácico Superior, se han definido varias asociaciones faunísticas de reptiles marinos a escala mundial (Massare, 1987; Bardet, 1995). Entre las más notables, pueden citarse las del Triásico Medio (Monte San Giorgio, Italia y Suiza) y del Jurásico Medio y Superior (Oxford Clay y Kimmeridge Clay en Inglaterra y niveles más o menos equivalentes en Francia) de Europa. Las faunas de reptiles marinos del Cretácico son principalmente conocidas gracias a los yacimientos norteamericanos. Así, las ricas faunas de vertebrados marinos del llamado "Western Interior Sea" (Santoniense-Campaniense) han sido estudiadas en detalle, tanto desde un punto de vista bioestratigráfico como paleobiogeográfico (Carpenter, 1990; Nicholls y Russell, 1990). Por el contrario, las faunas contemporáneas de vertebrados marinos de Europa no han recibido la atención que merecen. Para cubrir esta laguna, se ha realizado una revisión sistemática y bioestratigráfica de los grupos de reptiles marinos implicados (véase Bardet, 1995).

En este trabajo se analizan las faunas de reptiles marinos del Cretácico final de Europa durante el intervalo comprendido entre el Santoniense y el Maastrichtiense. No se han tenido en cuenta los restos de reptiles marinos del Turoniense y Coniaciense debido al carácter 


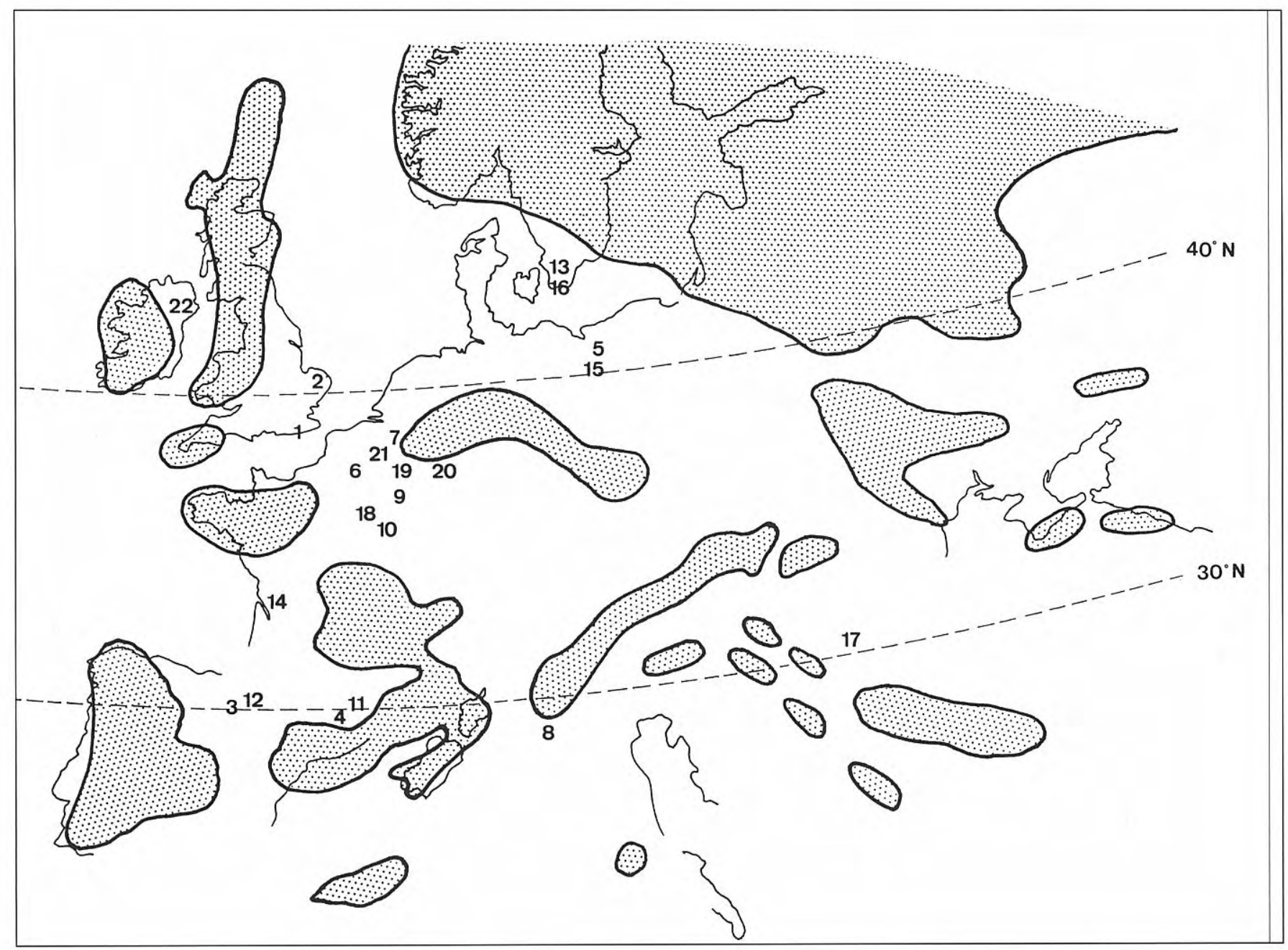

Figura 1. Mapa geográfico de Europa durante el Cretácico superior (Santoniense-Campaniense); basado en Dercourt et al. (1986) y Ziegler (1987). Los números hacen referencia a los yacimientos citados en el texto: Turoniense a Campaniense: 1. Houghton, Brighton y Offham, Sussex; 2. Norfolk. Santoniense: 3. San Pantaleón, Castilla; 4. Bugarach y Sougraigne, Languedoc; 5. Malbork, Prusia; 6. Vaux-Eclusier, Picardía; 7. Lonzée, Namur. Santoniense o Campaniense: 8. Zavattarello, Lombardía. Campaniense inferior: 9. Brimont, Champagne; 10. Michery, Borgoña; 11. La Bastide, Languedoc; 12. Castillo, País Vasco; 13. Isla de Ivö y otras localidades, Escania; 14. Mortagne-sur-Gironde, Gironda. Campaniense superior: 15. Malbork, Prusia; 16. Isla de Ivö, Escania; 17. Somovit y Vrbesnica, Bulgaria; 18. Meudon, Ile-de-France; 19. Harmignies, Henao; 20. Halden, Westfalia; 21. Ciply, Henao. Campaniense superior o Maastrichtiense inferior: 22. Whitewell, Irlanda del Norte.

fragmentario del registro fósil europeo. Los sedimentos turonienses han proporcionado principalmente restos fragmentarios de mosasaurios en el condado de Kent, Inglaterra (Woodward, 1905), de aigialosaurios en Croacia (Carroll y Debraga, 1992), de pliosaurios en Bohemia (Fritsch y Bayer, 1905) y posiblemente en Sussex (Owen, 1851), así como una tortuga marina en Turena, Francia (Bardet et al., en prensa). Otros restos incompletos de mosasaurios y de tortugas provienen del Coniaciense de Italia (Cigala-Fulgosi et al., 1980).

\section{MARCO ESTRATIGRÁFICO Y PALEOGEOGRÁFICO}

Durante el Cretácico Superior Europa se convirtió en un rosario de islas separadas unas de otras por un extenso mar epicontinental. Esta configuración estuvo controlada por movimientos complejos de las placas tectónicas en el dominio del mar de Tetis, durante un régimen global de tipo transgresivo (Dercourt et al., 1986; Haq et al., 1987; Ziegler, 1987). Las reconstrucciones palinspásticas más recientes sugieren que una gran transgresión tuvo lugar entre el Albiense y el Maastrichtiense, con una extensión máxima del mar epicontinental entre el Campaniense superior y el Maastrichtiense inferior (Tyson y Funnell, 1987). Los sedimentos marinos depositados durante esta época, principalmente calizas de plataforma, contienen una rica fauna de moluscos, foraminíferos y vertebrados fósiles. Entre estos últimos, los reptiles marinos ocupan un lugar importante. Varias formaciones geológicas han proporcionado una asociación faunística variada, siendo las más conocidas las que afloran en la Cuenca de Mons, en Bélgica, y en la Cuenca de Maastricht-Hesbaya, 


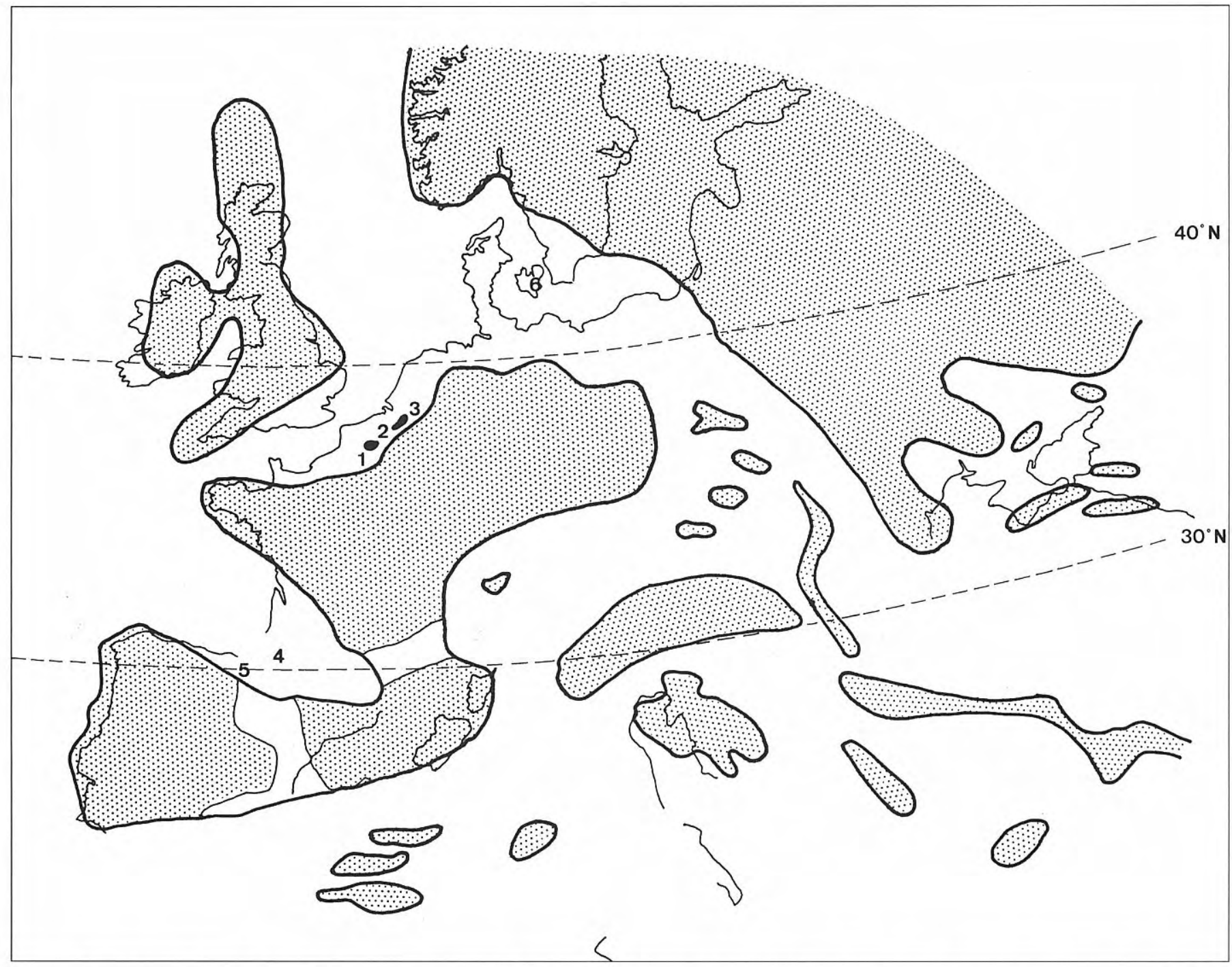

Figura 2. Mapa geográfico de Europa durante el Cretácico final (Maastrichtiense); basado en Camoin et al. (1993). Maastrichtiense inferior: 1. Ciply y otras localidades, Cuenca de Mons, Henao. Maastrichtiense superior: 2. Folx-les-Caves, Brabante; 3. Maastricht y otras localidades, Cuenca de Maastricht, Limburgo; 4. Cardesse, Bearne; 5. Laño, Condado de Treviño; 6. Stevns Klint, Sjaelland, Dinamarca.

situada entre Bélgica y Holanda. Además de las áreas citadas se conocen otras formaciones fosilíferas que han suministrado restos incompletos en Alemania, Bulgaria, Dinamarca, Francia, Inglaterra, Irlanda, Italia, Polonia, Suecia y la península Ibérica (Figs. 1-2). La rica fauna de moluscos ha permitido establecer una correlación bioestratigráfica basada principalmente en las zonas de belemnites (Schulz et al., 1984; Christensen, 1986, 1990) (Fig. 3). Esta biozonación ha servido de referencia para definir la distribución estratigráfica de las faunas de reptiles marinos (Fig. 4).

\section{ANÁLISIS SISTEMÁTICO}

Los reptiles marinos están representados en el Cretácico final de Europa por las tortugas, los mosasaurios y los plesiosaurios, estos últimos incluyendo pliosaurios y elasmosaurios. Las tortugas y sobre todo los mosasaurios son los grupos más diversificados, como sugieren el gran número de especies y de especímenes fósiles descubiertos. Así, 52 esqueletos de mosasaurios fueron excavados entre 1880 y 1895 , período de intensa explotación de las canteras, en la llamada "creta fosfatada" de Ciply, en la Cuenca de Mons, Henao, Bélgica (Lingham-Soliar y Nolf, 1989). Se han descrito varias decenas de especies de mosasaurios en Europa, pero sólo dieciocho de ellas, representando nueve géneros, son consideradas válidas en este trabajo. En lo que concierne a las tortugas, no menos de 257 especímenes fósiles han sido hallados en la región de Limburgo, situada entre Bélgica y Holanda, durante el período 1766-1978 (Felder, 1980). Sin embargo, la diversidad de las tortugas europeas es, desde un punto de vista sistemático, considerablemente menor que la de los mosasaurios y, de hecho, sólo pueden reconocerse actualmente cuatro géneros monoespecíficos (Bardet, 1995). Por último, los plesiosaurios son escasos en los 


\begin{tabular}{|c|c|c|c|c|c|c|}
\hline PISOS & & $\begin{array}{l}\text { BIOZ. BELEMNITES } \\
\text { NW ALEMANIA }\end{array}$ & $\begin{array}{l}\text { BIOZ. BELEMNITES } \\
\text { ESTANDAR EUROPA }\end{array}$ & $\begin{array}{c}\text { MONS } \\
\text { BELGICA }\end{array}$ & $\begin{array}{c}\text { HESBAYA-MAASTRICHT } \\
\text { HOLANDA-BELGICA }\end{array}$ & FRANCIA \\
\hline \multirow{3}{*}{$\begin{array}{l}\text { MAASTRICHTIENSE } \\
\text { SUPERIOR }\end{array}$} & $\mathrm{s}$ & baltica/danica & B. casimirovensis & & \multirow{2}{*}{ Fm. Maastricht } & \multirow{3}{*}{ Marnes de Nay } \\
\hline & \multirow[b]{2}{*}{1} & danica/argentea & \multirow[b]{2}{*}{ B. junior } & \multirow[b]{2}{*}{ Craie de Saint-Symphorien } & & \\
\hline & & $\begin{array}{l}\text { argentea/junior } \\
\text { tegulatus/junior }\end{array}$ & & & \multirow{10}{*}{ Fm. Gulpen } & \\
\hline \multirow{6}{*}{$\begin{array}{l}\text { MAASTRICHTIENSE } \\
\text { INFERIOR }\end{array}$} & \multirow{3}{*}{ s } & fastigata & B. fastigata & & & \\
\hline & & cimbrica & B. cimbrica & & & \\
\hline & & sumensis & B. sumensis & & & \\
\hline & \multirow{3}{*}{1} & obtusa & B. obtusa & Craie de Ciply & & \\
\hline & & pseudobtusa & B. pseudobtusa & & & \\
\hline & & lanceolata & B. lanceolata & & & \\
\hline \multirow{3}{*}{$\begin{array}{l}\text { CAMPANIENSE } \\
\text { SUPERIOR }\end{array}$} & \multirow[t]{2}{*}{ S } & $\begin{array}{c}\text { grimmensis/granulosus } \\
\text { langei }\end{array}$ & B. "langei" & $\begin{array}{l}\text { Craie de } \\
\text { Spiennes }\end{array}$ & & \\
\hline & & polyplocum/B."minor" & B. "minor" & & & \\
\hline & 1 & $\begin{array}{c}\text { vulgaris } \\
\text { stobaei/basiplana } \\
\text { conica/mucronata } \\
\end{array}$ & B. mucronata & $\begin{array}{c}\text { Craies de } \\
\text { Nouvelles \& d'Obourg }\end{array}$ & & Craie de Meudon \\
\hline \multirow{7}{*}{$\begin{array}{l}\text { CAMPANIENSE } \\
\text { INFERIOR }\end{array}$} & & gracilis/mucronata & G. quadrata gracilis & \multirow{12}{*}{$\begin{array}{l}\text { Glauconie de } \\
\text { Lonzée }\end{array}$} & & Craie de l'Yonne \\
\hline & $\mathrm{s}$ & conica/gracilis & & & & \\
\hline & & papillosa & \multirow{4}{*}{ G. quadrata quadrata } & & & \\
\hline & & $\frac{\text { senonensis }}{2 \text {. }}$ & & & & \\
\hline & \multirow{3}{*}{1} & $\begin{array}{c}\text { pilula/senonensis } \\
\text { pilula }\end{array}$ & & & & \\
\hline & & lingua/quadrata & & & & \\
\hline & & granulataquadrata & G. granulataquadrata & & & Grès de Labastide \\
\hline \multirow{5}{*}{ SANTONIENSE } & \multirow[t]{2}{*}{$S$} & Marsupites/granulata & \multirow[t]{2}{*}{ G. granulata } & & & Marnes de Sougraigne \\
\hline & & Uintacrinus/granulata & & & & \\
\hline & $M$ & westfalicagranulata & G. westfalicagranulata & & & Craie de la \\
\hline & & cordiformis/westfalica & G. westfalica westfalica & & & Somme \\
\hline & 1 & undulatoplicatus & & & & \\
\hline
\end{tabular}

Figura 3. Diagrama de correlación bioestratigráfica de las principales áreas fosilíferas del Cretácico final de Europa. Las columnas 1 y 2 (biozonas) están basadas en Schulz et al. (1984) y Christensen (1986, 1990, 1994), la columna 3 en Robaszynski y Christensen (1989) y Christensen (1994), la columna 4 en Schulz y Schmid (1983) y Bless et al. (1993), y la columna 5 ha sido elaborada a partir de Bardet et al. (1991), Christensen et al. (1993) y los datos de la Carte géologique de la France au $1 / 50000\left(n^{\circ} 47,108,183,295,1051\right)$.

sedimentos del Cretácico final de Europa. Tanto los restos de pliosaurios como de elasmosaurios son muy fragmentarios y de poco valor diagnóstico, de modo que ningún taxón de rango inferior a la familia puede ser considerado válido (Bardet y Godefroit, 1995).

Por otro lado, dos grupos de reptiles marinos conocidos en el registro fósil durante el Cretácico final no aparecen representados en los yacimientos europeos. Se trata de los cocodrilos (Dyrosauridae) y las serpientes (Paleophidae). Su ausencia en los yacimientos europeos puede obedecer a causas ecológicas pero no debe descartarse que se trate de un artefacto tafonómico (véase discusión).

\section{Testudines}

En el curso de su historia, varios grupos de tortugas se han adaptado a un modo de vida marino. Durante el Cretácico, se conocen una familia de tortugas litorales (Bothremydidae) y varias familias de tortugas pelágicas, todas ellas incluídas dentro de la superfamilia Chelonioidea (Zangerl, 1980). Algunas de estas familias, como las Cheloniidae y Dermatochelyidae, cuentan todavía con representantes en la actualidad.

La posición sistemática de las tortugas marinas del Cretácico final de Europa es problemática. Por un lado, las especies Glaucochelone lonzeensis y Tomochelone lonzeensis, del Santoniense (Fm. Glauconie de Lonzée) de Bélgica, y Platychelone emarginata, del Maastrichtiense (Fm. Maastricht) de Holanda, fueron citadas por Dollo (1909), pero el material no ha sido estudiado en detalle ni ilustrado. En este trabajo, consideramos válida la última especie siguiendo la opinión de Felder (1980). Por otro lado, la validez sistemática de Allopleuron hoffmanni (Gray, 1831) y Glyptochelone suyckerbuyki (Ubaghs, 1879), del Maastrichtiense de Bélgica y Holanda, no ofrece dudas. No obstante, debido a que estas especies no han sido nunca debidamente revisadas, su atribución familiar es confusa y varía dependiendo de los autores. Así, Allopleuron es considerado como un miembro de la familia Cheloniidae (Mlynarski, 1976; Zangerl, 1980) o Desmatochelyidae (Moody, 1993). Según Gaffney y Meylan (1988), Allopleuron comparte con Desmatochelys varios caracteres derivados y ambos a su vez están más emparentados con los Dermochelyoidea que con las otras tortugas. En este trabajo, Allopleuron, Glyptochelone y Platychelone son considerados provisionalmente como Chelonioidea incertae sedis. Numerosos especímenes de Allopleuron hoffmanni provienen de las formaciones maastrichtienses de las cuencas de Mons (Henao) y Maastricht (Limburgo). La distribución estratigráfica del género podría extenderse hasta el Santoniense, a raíz del descubrimiento de restos fragmentarios en la Fm. de Losas, Burgos (Bardet et al., 1993). La especie Glyptochelone suyckerbuyki ha sido 


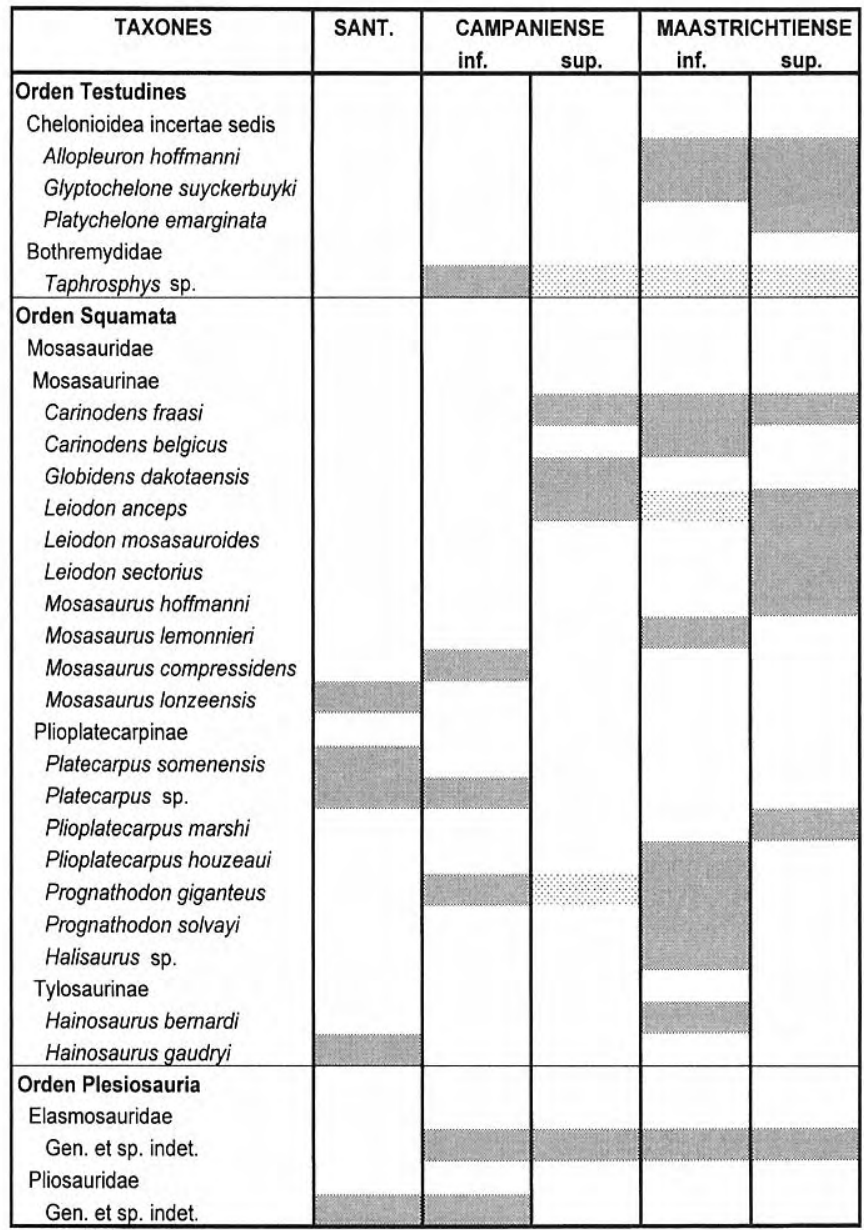

Figura 4. Distribución estratigráfica de las faunas de reptiles marinos del Cretácico final (Santoniense a Maastrichtiense) de Europa. La trama oscura indica la distribución conocida y la trama clara los "taxones lázaro".

hallada en los mismos niveles estratigráficos que Allopleuron hoffmanni, mientras que Platychelone emarginata es conocida únicamente en el Maastrichtiense superior de la Cuenca de Maastricht (Felder, 1980).

Persson (1959) menciona Osteopygis? sp. en el Campaniense de Suecia, pero la presencia de la familia Osteopygidae en el Cretácico final de Europa es dudosa. Por otra parte, la familia Bothremydidae es conocida en el Cretácico final de Europa a partir de formas de agua dulce y del género litoral Taphrosphys, éste último descubierto en sedimentos lagunares del Campaniense inferior (Fuveliense) de Coudoux, Provenza (Antunes y Broin, 1988).

\section{Squamata}

Los mosasaurios, lagartos varanoides bien adaptados al medio acuático, constituyeron un componente importante de los ecosistemas marinos del Cretácico Superior (Russell, 1967). En un contexto histórico, el estudio de los mosasaurios está bien arraigado en la paleontología europea ya que los primeros especímenes descritos en la literatura fueron descubiertos durante la segunda mitad del siglo XVIII (Bardet y Jagt, en prensa).
Sistemáticamente, los Mosasauridae son el grupo hermano de los Aigialosauridae, reptiles acuáticos hallados en sedimentos del Cenomaniense-Turoniense de Istria y Dalmacia (Carroll y Debraga, 1992). El registro más antiguo de mosasaurios proviene del Turoniense, pero sus restos sólo empiezan a ser abundantes en los yacimientos a partir del Santoniense. La familia Mosasauridae incluye tres subfamilias (Mosasaurinae, Plioplatecarpinae y Tylosaurinae) y cada una de ellas cuenta con representantes en el registro fósil europeo.

Los restos de mosasaurios del Santoniense de Europa provienen fundamentalmente de Bélgica y Francia. La Fm. Glauconie de Lonzée (provincia de Namur, Bélgica) ha proporcionado al menos dos especies de mosasaurios, asignados a Mosasaurus lonzeensis y Hainosaurus sp. (Dollo, 1904; Bardet, 1990). En Francia, los restos descritos originalmente por Thevenin (1896) proceden de la Fm. Craie brune phosphatée de Picardía. Se trata de Hainosaurus gaudryi y Platecarpus somenensis (Bardet, 1990; Lingham-Soliar, 1992). Otros especímenes, hallados en las formaciones Marnes de Bugarach y Marnes de Sougraigne de Languedoc, son atribuidos provisionalmente a Platecarpus sp., pero podrían pertenecer a una nueva especie (Sénesse, 1936; Bardet et al., 1991).

El Campaniense de Europa ha proporcionado restos de mosasaurios en Alemania, Bélgica, Bulgaria, Francia, Inglaterra, Irlanda, País Vasco y Suecia. Se han identificado los géneros Carinodens, Globidens, Leiodon, Mosasaurus, Platecarpus y Prognathodon, pero sólo en ciertos casos es posible hacer una determinación específica. En Francia, Mosasaurus compressidens proviene de la Fm. Craie blanche de Yonne, en Borgoña y de la Fm. Calcaire de Coquand de Gironda (Gaudry, 1892), y Prognathodon giganteus de la Fm. Craie de Reims de Champagne (Bardet et al., en prep.). Leiodon anceps y Mosasaurus sp. se conocen en la Fm. Craie de Meudon, Ile-de-France (Hébert, 1855) y Platecarpus sp. en la Fm. Grès de Labastide, Languedoc (Bardet et al., 1991). En Bélgica, la Fm. Craie d'Obourg de la Cuenca de Mons ha proporcionado Globidens dakotaensis (Dollo, 1913; Russell, 1975) y posiblemente Leiodon sp. (Dollo, 1924; Lingham-Soliar, 1993). Leiodon anceps, Carinodens fraasi y Mosasaurus sp. han sido descritos en el Campaniense (Zona de Mucronata) del norte de Bulgaria (Tzankov, 1939; Nikolov y Westphal, 1976). Otros restos de mosasaurios, principalmente dientes y vértebras aislados, han sido descritos en la creta campaniense de Inglaterra (Sussex, Kent, Surrey, Hampshire, Wiltshire y Norfolk) e Irlanda del Norte (véase Owen, 1851; Jukes-Browne y Hill, 1904; Milner, 1987; Benton y Spencer, 1995) pero este material no ha sido revisado en detalle. Según Milner (1987) las tres subfamilias de mosasaurios están representadas en Inglaterra. Leiodon anceps y Mosasaurus sp. son conocidos en la Fm. Upper Chalk (datada del Turoniense superior-Campaniense superior, según Rawson et al., 1978) de Norfolk, Essex, Surrey y la costa de Sussex, Inglaterra (Owen, 1851; Lingham-Soliar, 1993). En Suecia, restos de mosasaurios atribuidos a los géneros 
Mosasaurus, Leiodon y Platecarpus provienen de los sedimentos campanienses (Zonas de Mammillatus y Mucronata) de la provincia de Escania (Persson, 1959). Otro material fragmentario atribuido a Mosasaurus sp. procede de la Fm. de Vitoria en Álava, País Vasco (Bardet et al., 1993) y de la Fm. White Limestones de Irlanda del Norte (Swanston, 1886). El género Mosasaurus podría también estar representado en el Campaniense (Zona de Polyplocum) de Westfalia, en Alemania (Pompeckj, 1910).

La asociación más importante de mosasaurios europeos proviene de los sedimentos maastrichtienses de las cuencas de Mons (Fm. Craie phosphatée de Ciply y Fm. Craie de Saint-Symphorien), en Henao, Bélgica, y Maastricht-Hesbaya (Fm. Gulpen y Fm. Maastricht), en Limburgo, Holanda. Al menos ocho especies son conocidas en la Fm. Craie de Ciply, de edad Maastrichtiense inferior: Carinodens belgicus, C. fraasi, Hainosaurus bernardi, Mosasaurus lemmonieri, Plioplatecarpus houzeaui, Prognathodon giganteus, $P$. solvayi y Halisaurus sp. (Dollo, 1924; Lingham-Soliar y Nolf, 1989). Por su parte, las formaciones Gulpen y Maastricht, de edad Maastrichtiense superior, han proporcionado Carinodens fraasi, Mosasaurus hoffmanni, Plioplatecarpus marshi (Dollo, 1924) y Leiodon sectorius (Lingham-Soliar, 1993). Otros restos asignados a Carinodens fraasi provienen de la Fm. Craie de Saint-Symphorien, del Maastrichtiense superior de Bélgica (Dollo, 1924). En el dominio iberooccitano, se han descrito restos de Leiodon mosasauroides en la Fm. Marnes de Nay, del Maastrichtiense superior del Bearne (Gaudry, 1892). Además, material inédito de Leiodon anceps y Mosasaurus sp. ha sido recientemente descubierto en el Maastrichtiense de Laño, Condado de Treviño (Bardet et al., en prep.). Se conocen también restos indeterminados de mosasaurios en el Maastrichtiense superior de Stevns Klint, Dinamarca (E. Hoch, com. pers.).

\section{Sauropterygia}

Durante el Cretácico Superior los plesiosaurios están representados por las familias Pliosauridae y Elasmosauridae, ambas conocidas en Europa (Brown, 1981). Los Elasmosauridae, presentes en el registro fósil desde el Liásico inferior, alcanzaron una gran diversidad de formas durante el Cretácico terminal. Se conocen numerosas especies que provienen de casi todos los continentes, lo que evidencia el carácter cosmopolita de la familia. No obstante, los sedimentos del Cretácico final de Europa ha proporcionado únicamente restos fragmentarios y poco diagnósticos. De hecho, todas las especies descritas en la literatura pueden ser consideradas nomina dubia (Bardet y Godefroit, 1995). Se han descrito restos de elasmosaurios en los depósitos de calizas ("chalk"), de edad Cenomaniense a Campaniense superior, de Sussex, en Inglaterra (Owen, 1851), en el Campaniense de Bélgica (Fm. Craie de Spiennes; Bardet y Godefroit, 1995), Suecia (Zona de Mammillatus; Persson, 1959) y Polonia (Zona de Mucronata; Schröder, 1885), en el Maastrichtiense de Bélgica (Fm. Tuffeau de
Folx-les-Caves; Meyer, 1860) y Holanda (Fm. Maastricht; Mulder, 1985, 1990).

Los Pliosauridae son conocidos en el registro fósil desde el Triásico terminal hasta el Cretácico final, pero su época de mayor esplendor fue el Jurásico Medio y Superior. Durante el Cretácico Superior la diversidad de los pliosaurios declinó, quizás debido a una competencia ecológica con los mosasaurios (Massare, 1987). A finales del Cretácico los pliosaurios están únicamente representados por restos muy escasos y fragmentarios. El Cretácico Superior de Europa ha proporcionado varios especímenes de pliosaurio, pero sólo el género Polyptychodon, del Cenomaniense-Turoniense de Inglaterra, Baviera y Bohemia, puede ser considerado válido (Bardet y Godefroit, 1995). Restos indeterminados a nivel genérico han sido descritos en la Fm. Upper Chalk (Turoniense superior-Campaniense superior) de Inglaterra (Owen, 1851), en el Santoniense de Polonia (Zona de Westphalica; Schröder, 1885) y Bélgica (Fm. Glauconie de Lonzée; Dollo, 1909), el SantonienseCampaniense de Lombardía (Fm. Argille Varicolori; Renesto, 1993) y el Campaniense inferior de Escania (Zona de Mammillatus; Persson, 1959).

\section{DISCUSIÓN}

Las faunas de reptiles marinos del Cretácico final están principalmente representadas por los mosasaurios, las tortugas y los plesiosaurios elasmosaurios. Los pliosaurios suponen un componente menor de las faunas, mientras que los ictiosaurios están ausentes de las mismas ya que se extinguen en la base del Cretácico Superior (Bardet, 1992).

La proporción relativa de cada uno de los grupos de reptiles marinos varía según los yacimientos. Así, en Norteamérica, los estudios realizados sobre el modelo de distribución de las faunas de vertebrados marinos (principalmente los reptiles) del llamado "Western Interior Sea" han revelado la existencia de dos provincias faunísticas en función de la paleolatitud durante el Santoniense-Campaniense (Nicholls y Russell, 1990). La paleoprovincia del norte está caracterizada por su baja diversidad específica y dominada por los plesiosaurios, las aves hesperornitiformes y el mosasaurio Platecarpus. Por su parte, la paleoprovincia del sur muestra una alta diversidad específica y está caracterizada por la abundancia de tiburones, tortugas y del mosasaurio Clidastes (Nicholls y Russell, 1990). Este modelo paleobiogeográfico apoya la hipótesis de una subdivisión del "Western Interior Sea" en una zona templada-fría septentrional y una zona templada-cálida meridional, cuyo límite latitudinal se situaría a unos $35^{\circ} \mathrm{N}$. Esta provincialización ha sido previamente sugerida por otros autores basándose en las faunas de invertebrados (p. ej. Kauffman, 1984).

Con respecto a las faunas de reptiles marinos del Cretácico final de Europa, los datos son insuficientes para conocer si existió una biozonación faunística similar a la comentada del "Western Interior Sea" norteamericano. 
Esta falta de información está probablemente provocada por la ausencia de megayacimientos, con excepción de las localidades de las cuencas de Mons y MaastrichtHesbaya. En primer lugar, hay que señalar que la geografía de Europa durante el Cretácico final, convertida en un archipiélago en el margen norte del mar de Tetis mediterráneo, es muy diferente de la del mar interior del oeste de Norteamérica. Las faunas europeas están globalmente dominadas por los mosasaurios y las tortugas, siendo los plesiosaurios menos frecuentes. Por otra parte, la mayoría de los géneros de mosasaurios europeos son conocidos en los yacimientos norteamericanos, sugiriendo una distribución cosmopolita, mientras que las tortugas son características de las faunas finicretácicas europeas (Bardet, 1995). Como ya se ha dicho anteriormente, los restos de elasmosaurios son indeterminados a nivel específico. No obstante, hay que precisar que las localidades más septentrionales, Escania y Prusia por ejemplo, han proporcionado más especímenes de plesiosaurios que de mosasaurios y tortugas, en comparación con los yacimientos situados más al sur, entre las paleolatitudes $40^{\circ} \mathrm{N}$ y $30^{\circ} \mathrm{N}$. Por último, los cocodrilos Dyrosauridae y las serpientes Paleophidae se conocen principalmente en los yacimientos situados sobre el margen sur del Tetis y el Golfo sahariano (Buffetaut, 1982; Rage, 1984). Su ausencia en la provincia europea puede haber estado controlada por las corrientes de dirección E-W del mar de Tetis, que habrían actuado como una barrera limitando su dispersión (Buffetaut, 1982). Otra hipótesis de orden ecológico es propuesta en este trabajo: los dirosáuridos y paleófidos preferían los ambientes más cálidos situados cerca del ecuador, lo que explica su frecuente presencia entre las paleolatitudes $20^{\circ} \mathrm{N}$ y $20^{\circ} \mathrm{S}$.

\section{CONCLUSIONES}

Los datos disponibles sobre las faunas de reptiles marinos del Cretácico final de la provincia europea (margen norte del mar de Tetis mediterráneo) permiten formular varias hipótesis de trabajo. En primer lugar, el hecho de que los plesiosaurios estén raramente asociados con los mosasaurios y las tortugas en los yacimientos podría indicar una provincialización según el gradiente paleolatitudinal, como sugieren Nicholls y Russell (1990). No obstante, no puede excluirse que este modelo de distribución esté condicionado por diferencias paleoambientales. En ese caso, es necesario realizar un estudio sedimentológico detallado de los sedimentos fosilíferos antes de pronunciarse en favor de la segunda posibilidad.

La asociación faunística de Henao y Limburgo está dominada por los mosasaurios y las tortugas y puede ser comparada a grandes rasgos con la de la provincia sur del "Western Interior Sea" de Norteamerica. Por su parte, la asociación faunística de Suecia y Prusia, aunque basada en restos incompletos, se asemeja más a la provincia norte del "Western Interior Sea", definida por Nicholls y Russell (1990). Por último, los cocodrilos dirosáuridos y las serpientes paleófidas están ausentes en las faunas de reptiles marinos del margen norte del mar de Tetis mediterráneo y su ausencia podría obedecer a causas de tipo ecológico.

Aunque preliminar, este trabajo de síntesis permite poner de manifiesto el interés de los yacimientos finicretácicos en facies marinas de Europa y su importancia a la hora de establecer una correlación con otras localidades, con vistas a la elaboración de un modelo global sobre la evolución de las faunas de vertebrados marinos de finales del Mesozoico.

\section{AGRADECIMIENTOS}

Los autores agradecen a Pascal Godefroit (Bruselas), John Jagt (Maastricht) y Eric Mulder (Denekamp) las informaciones y referencias sobre la estratigrafía de Bélgica y Holanda. Nuestro agradecimiento va también dirigido a Zulma Gasparini (La Plata) y José Luis Sanz (Madrid) por la revisión crítica del manuscrito. Un resumen de este trabajo fue presentado durante las VI Jornadas Argentinas de Paleontología y Bioestratigrafía celebradas en Trelew (Chubut) en 1994 gracias a una ayuda de la Fundación Singer-Polignac de París.

\section{BIBLIOGRAFÍA}

Antunes, M.T. et Broin, F. de 1988. Le Cretacé terminal de Beira Litoral, Portugal: remarques stratigraphiques et écologiques, étude complémentaire de Rosasia soutoi (Chelonii, Bothremydidae). Ciências da Terra, 9, 153200.

Bardet, N. 1990. Première mention du genre Hainosaurus (Squamata, Mosasauridae) en France. Comptes Rendus de l'Academie des Sciences, II, 311, 751-756.

Bardet, N. 1992. Stratigraphic evidences for the extinction of ichthyosaurs. Terra Nova, 4, 649-656.

Bardet, N. 1995. Evolution et extinction des reptiles marins au cours du Mésozoïque. Palaeovertebrata, 24 (3-4).

Bardet, N. and Godefroit, P. 1995. Plesiosaurus houzeaui DOLLO, 1909 from the Upper Campanian of Ciply (Belgium) and a review of the Upper Cretaceous plesiosaurs from Europe. Bulletin de l'Institut Royal des Sciences Naturelles de Belgique, Sciences de la Terre, 65, 179-186.

Bardet, N. et Jagt, J.W.M. (en prensa). Mosasaurus hoffmanni, le "Grand Animal Fossile des Carrières de Maestricht": deux siècles d'histoire. Bulletin du Museum national d'histoire naturelle, sect. C, 18 (2).

Bardet, N., Bilotte, M. et Christensen, W.K. 1991. Nouveaux restes et révisions de faunes exceptionnelles du Crétacé supérieur audois: les Bellemnitellidae et Mosasauridae des Corbières. Bulletin de la Société d'Etudes scientifiques de l'Aude, 91, 11-21.

Bardet, N., Corral, J.C. y Pereda Suberbiola, X. 1993. Primeros restos de reptiles marinos en el Cretácico superior de la Cuenca Vasco-Cantábrica. Estudios del Museo de Ciencias Naturales de Alava, 8, 27-35. 
Bardet, N., Pereda Suberbiola, X. and Badillet, G. (en prensa). A primitive chelonioid turtle from the Late Cretaceous of Touraine, France. Bulletin de la Société géologique de France, 167 (2).

Benton, M.J. and Spencer, P.S. 1995. Fossil Reptiles of Great Britain. In: Geological Conservation Review Series (Eds. W.A. Wimbledon and D. Palmer), 10, 386 pp. Chapman \& Hall, Londres.

Bless, M.J.M., Dusar, M., Felder, P.J. and Swennen, R. 1993. Lithology and biostratigraphy of Upper Cretaceous-Paleocene carbonates in the Molenbeersel borehole (NE Belgium). Geologie en Mijnbouw, 71, 239257.

Brown, D.S. 1981. The English Upper Jurassic Plesiosauroidea (Reptilia) and a review of the phylogeny and classification of the Plesiosauria. Bulletin of the British Museum (Natural History), Geology, 35 (4), 253347.

Buffetaut, E. 1982. Radiation évolutive, paléoécologie et biogéographie des crocodiles mésosuchiens. Mémoires de la Société géologique de France, N.S., 60 (142), 1-80.

Camoin, G., Bellion, Y., Dercourt, J., Guiraud, R., Lucas, J., Poisson, A., Ricou, L.E. and Vrielynck, B. 1993. Late Maastrichtian palaeoenvironments (69,5 to $65 \mathrm{Ma})$. In: Atlas Tethys Palaeonvironmental Maps (Eds. J. Dercourt, L.E. Ricou \& B. Vrielynck). BEICIPFRANLAB, Rueil-Malmaison, 179-196.

Carpenter, K. 1990. Upward continuity of the Niobrara fauna with the Pierre Shale fauna. In: Niobrara Chalk Excursion Guidebook (Ed. S.C. Bennett). Society of Vertebrate Paleontology, University of Kansas Museum Natural History \& Kansas Geological Survey, 73-81.

Carroll, R.L. and Debraga, M. 1992. Aigialosaurs: midCretaceous varanoid lizards. Journal of Vertebrate Paleontology, 12 (1), 66-86.

Christensen, W.K. 1986. Upper Cretaceous belemnites from the Vomb Trough in Scania, Sweden. Sveriges Geologiska Undersökning, ser. Ca, 57, 1-57.

Christensen, W.K. 1990. Upper Cretaceous belemnite stratigraphy of Europe. Cretaceous Research, 11 (4), 371-386.

Christensen, W.K. 1994. Upper Cretaceous belemnites from Lonzée (SE Belgium) and their stratigraphical significance. Bulletin de l'Institut Royal des Sciences Naturelles de Belgique, Sciences de la Terre, 64, 151158.

Christensen, W.K., Bilotte, M. and Hansotte, M. 1993. Additional Late Cretaceous belemnitellids from the Corbières, French Pyrenees and ammonite biostratigraphy of the Santonian. Cretaceous Research, 14, 101-106.

Cigala-Fulgosi, F., Kotsakis, T., Massari, F., Medizza, F. e Sorbini, L. 1980. Il giacimento di S. Anna d'Alfaedo. In: I vertebrati fossili italiani, Catalogo della Mostra, Verona, 123-129.

Dercourt, J., Zonenshain, L.P., Ricou, L.-E., Kazmin, V.G., Le Pichon, X., Knipper, A.L., Grandjacquet, C., Sbortshikov, J.M., Geyssant, J., Lepvrier, C., Pechersky, D.H., Boulin, J., Sibuet, J.-C., Savostin, L.A., Sorokhtin, O., Westphal, M., Bazhenov, M.L., Lauer, J.P. and Biju-
Duval, B. 1986. Geological evolution of the Tethys belt from the Atlantic to the Pamir since the Lias. In: Evolution of the Tethys (Eds. J. Auboin, X. Le Pichon. \& A.S Monin). Tectonophysics, 123, 241-315. .

Dollo, L. 1904. Les mosasauriens de la Belgique. Bulletin de la Société Belge de Géologie, de Paléontologie et d'Hydrologie, 18, 207-216.

Dollo, L. 1909. The fossil vertebrates of Belgium. Annals of the New York Academy of Sciences, 19, 4 (1), 99-119.

Dollo, L. 1913. Globidens fraasi, mosasaurien mylodonte nouveau du Maestrichtien (Crétacé supérieur) du Limbourg, et l'éthologie de la nutrition chez les Mosasauriens. Archives de Biologie, 28, 609-626.

Dollo, L. 1924. Globidens alabamaensis, mosasaurien mylodonte américain retrouvé dans la Craie d'Obourg (Sénonien supérieur) du Hainaut, et les Mosasauriens de la Belgique, en général. Archives de Biologie, 34, 167-213.

Felder, P.J. 1980. Resten van fossiele zeeschildpadden gevonden in het Krijt van Limburg. Natuurhistorisch Maandblad, 69 (5), 100-104.

Fritsch, A. und Bayer, F. 1905. Neue Fische und Reptilien aus der böhmischen Kreideformation. Praga, 1-34.

Gaffney, E.S. and Meylan, P.A. 1988. A phylogeny of turtles. In: The Phylogeny and Classification of the Tetrapods, vol. 1: Amphibians, Reptiles, Birds (Ed. M.J. Benton). Systematic Association, spec. vol., 35A, 157219. Clarendon Press, Oxford.

Gaudry, A. 1892. Les Pythonomorphes de France. Mémoires de la Société géologique de France, 10, 1-13.

Gray, J.E. 1831. Synopsis Reptilium; or, short descriptions of the species of Reptiles. Synopsis Reptilium, Part I: Tortoises, Crocodiles and Enaliosaurians, 3, 1-85.

Haq, B.U., Handerbol, J. and Vail, P.R. 1987. Chronology of fluctuating sea levels since the Triassic. Science, 235, 1156-1167.

Hébert, E. 1855. Tableau des fossiles de la Craie de Meudon. Mémoires de la Société géologique de France, II, 5 (4), 345-374.

Jukes-Browne, A.J. and Hill, W. 1904. The Cretaceous Rocks of Britain. Vol. III. The Upper Chalk of England. Memoirs of the Geological Survey of the United Kingdom, Londres, 566 pp.

Kauffman, E.G. 1984. Paleobiogeography and evolutionary response dynamic in the Cretaceous Western Interior Seaway of North America. In: Jurassic-Cretaceous Paleogeography of North America (Ed. G.E.G. Westermann). Geological Association of Canada, spec. paper, 27, 273-306.

Lingham-Soliar, T. 1992. The tylosaurinae mosasaurs (Reptilia, Mosasauridae) from the Upper Cretaceous of Europe and Africa. Bulletin de l'Institu Royal des Sciences Naturelles de Belgique, Sciences de la Terre, 62, 171-194.

Lingham-Soliar, T. 1993. The Mosasaur Leiodon bares its teeth. Modern Geology, 18, 443-458.

Lingham-Soliar, T. and Nolf, D. 1989. The mosasaur Prognathodon (Reptilia, Mosasauridae) from the Upper Cretaceous of Belgium. Bulletin de l'Institut Royal des Sciences Naturelles de Belgique, Sciences de la Terre, 59, 137-190. 
Massare, J.A. 1987. Tooth morphology and prey preference of Mesozoic marine reptiles. Journal of Vertebrate Paleontology, 7 (2), 121-137.

Meyer, H. von 1860. Saurier aus der Tuff-Kreide von Maastricht und Folx-les-Caves. Palaeontographica A, 7, 241-244.

Milner, A.C. 1987. Reptiles. In: Fossils of the Chalk (Ed. A.B. Smith). Palaeontological Association Field Guides to Fossils, 2, 266-280. The Palaeontological Association, Londres.

Mlynarski, M. 1976. Testudines. In: Handbuch der Paläoherpetologie (Ed. O. Kuhn), 19, 1-130. Gustav Fisher, Stuttgart y Nueva York.

Moody, R.T.J. 1993. Cretaceous-Tertiary Marine turtles of the North West Europe. Revue de Paléobiologie, vol. spéc. 7, 151-160.

Mulder, E.W.A. 1985. Plesiosauriërs in de Limburgse Krijtzee. Natuurhistorisch Maandblad, 74 (5), 94-96.

Mulder, E.W.A. 1990. Ein Elasmosaurierzahn aus der oberen Kreide des St. Pietersberges bei Maastricht, SüdLimburg, Niederlande. Paläontologische Zeitschrift, 64 (1/2), 145-151.

Nicholls, E.L. and Russell, A.P. 1990. Paleobiogeography of the Cretaceous Western Interior Seaway of North America: the vertebrate evidence. Palaeogeography, Palaeoclimatatology, Palaeoecolology, 79, 149-169.

Nikolov, I. und Westphal, F. 1976. Mosasaurier-Funde aus der Oberkreide von Nordwest-Bulgarien. Neues Jarhbuch für Geologie und Paläontologie Mh., 1976 (10), 608-613.

Owen, R., 1851. Monograph on the Fossil Reptilia of the Cretaceous Formations. Monographs of the Palaeontographical Society of London, 5 (11), 1-118.

Persson, P.O. 1959. Reptiles from the Senonian (Upper Cretaceous) of Scania (S. Sweden). Arkiv för Mineralogi och Geologi, 2 (35), 431-478.

Pompeckj, J.F. 1910. Über einen Fund von MosasaurierResten im Ober-Senon von Haldem. Jahresbericht des Niedersächsischen geologischen Vereins, 3, 122-140.

Rage, J.-C. 1984. Serpentes. In: Handbuch der Paläoherpetologie (Ed. P. Wellnhofer), 11, xi +80 . Gustav Fisher, Stuttgart y Nueva York.

Rawson, P.F., Curry, D., Dilley, F.C., Hancock, J.M., Kennedy, W.J., Neale, J.W., Wood, C.J. and Worssam, B.C. 1978. A correlation of Cretaceous rocks in the British Isles. Geological Society of London, spec. rep. 9, $1-70$.

Renesto, S. 1993. A Cretaceous plesiosaur remain (Reptilia, Sauropterygia) from the Argille Varicolori de Varzi (Pavia, Lombardy, Northern Italy). Rivista Italiana di Paleontologia e Stratigrafia, 99 (1), 101-106.

Robaszynski, F. and Christensen, W.K. 1989. The Upper Campanian-Lower Maastrichtian chalks of the Mons basin, Belgium: a preliminary study of belemnites and foraminifera in the Harmignies and Ciply areas. Geologie en Mijnbouw, 68, 391-408.

Russell, D.A. 1967. Systematics and Morphology of the American Mosasaurs. Bulletin of the Peabody Museum of Natural History, Yale University, 23, 1-241.

Russell, D.A. 1975. A new species of Globidens from South Dakota, and a review of Globidentine Mosasaurs. Fieldiana Geology, 33 (13), 235-256.

Schröder, H. 1885. Saurierreste aus der baltischen oberen Kreide. Jahrbuch der preussischen geologischen Landesanstalt und Bergakademie, 5 (1884), 293-333.

Schulz, M.-G. und Schmid, F. 1983. Das Ober-Maastricht von Hemmoor (N-Deutschland): FaunenzonenGliederung und Korrelation mit dem Ober-Maastricht von Dänemark und Limburg. Newsletters on Stratigraphy, 13 (1), 21-39.

Schulz, M.-G., Ernst, G., Ernst, H. and Schmid, F. 1984. Coniacian to Maastrichtian stage boundaries in the standard section for the Upper Cretaceous white chalk of NW Germany (Lägerdorf-Kronsmoor-Hemmoor): Definition and proposals. Bulletin of the geological Society of Denmark, 33 (1/2), 203-215.

Sénesse, P. 1936. Le Mosasaure des Corbières. Impr. Basuyau, Toulouse, 1-15.

Swanston, W. 1886. Mosasaurus gracilis, Owen from the Irish Chalk. Proceedings \& Reports of the Belfast Natural History and Philosophical Society, 1886, 134.

Thevenin, A. 1896. Mosasauriens de la Craie grise de VauxEclusier près Péronne (Somme). Bulletin de la Société géologique de France, III, 24, 900-916.

Tyson, R.V. and Funnell, B.M. 1987. European Cretaceous shorelines, stage by stage. Palaeogeography, Palaoeclimatology, Palaeoecology, 59, 69-91.

Tzankov, V. 1939. Note sur la présence de reptiles fossiles du Crétacé supérieur de la Bulgarie du Nord. Geologica Balkanica, 3 (2), 13-20.

Ubaghs, C. 1879. Description géologique et paléontologique du sol du Limburg, avec catalogue général des fossiles du terrain crétacé, coupe de la superposition des couches et description de quelques grands vertébrés de la craie supérieure de Maastricht. Romen et fils, Ruremonde, ii +275 p.

Woodward, A.S. 1905. Note on some portions of mosasaurian jaws obtained by Mr. G. E. Dibley from the Middle Chalk of Cuxton, Kent. Proceedings of the Geologists' Association, 19 (4), 185-187.

Zangerl, R. 1980. Patterns of phylogenetic differenciation in the toxochelyid and cheloniid sea turtles. American Zoology, 20, 585-596.

Ziegler, P.A. 1987. Late Cretaceous and Cenozoic intraplate compressional deformations in the Alpine foreland - a geodynamic model. Tectonophysics, 137, 389-420. 